\title{
Conhecimento sobre câncer de mama entre enfermeiros da atenção primária de Divinópolis/MG
}

RESUMO | Objetivo: avaحliação do nível de conhecimento dos enfermeiros da atenção primária da rede pública de Divinópolis (MG) sobre o câncer de mama. Método: pesquisa quantitativa, descritiva, de delineamento transversal com profissionais de enfermagem, que atuavam há pelo menos um ano na rede municipal de saúde. Foi utilizado questionário estruturado, analisada a distribuição das variáveis de interesse e para apresentação dos mesmos, utilizou-se a estatística descritiva, média, frequência absoluta e porcentagem. Resultado: Foi identificado o conhecimento pelos entrevistados, sendo a mamografia o exame mais indicado para diagnóstico precoce, porém foi identificado duvidas na idade e periodicidade recomendada para realização do exame, e os fatores limitantes ao rastreamento mais referidos foram o desconhecimento da população e o equipamento insuficiente. Conclusão: Fazem-se necessários a abordagem do tema no período de graduação e de educação continuada, investimento na capacitação dos profissionais e ampliação da prevenção da doença e promoção da saúde.

Palavras-chaves: Neoplasias da Mama; Enfermeiros; Conhecimentos.

\begin{abstract}
Objective: to assess the level of knowledge of nurses in primary care in the public network of Divinópolis (MG) about breast cancer. Method: quantitative, descriptive, cross-sectional research with nursing professionals, who had worked for at least one year in the municipal health system. A structured questionnaire was used, the distribution of the variables of interest was analyzed and for their presentation, descriptive statistics, mean, absolute frequency and percentage were used. Result: Knowledge was identified by the interviewees, with mammography being the most suitable exam for early diagnosis, but doubts were identified in the age and recommended periodicity for the exam, and the most mentioned limiting factors for screening were the lack of knowledge of the population and the equipment insufficient. Conclusion: It is necessary to approach the theme in the period of graduation and continuing education, investment in the training of professionals and expansion of disease prevention and health promotion.
\end{abstract}

Keywords: Breast Neoplasms; Nurses; Knowledge.

RESUMEN | Objetivo: evaluar el nivel de conocimiento de las enfermeras en atención primaria en la red pública de Divinópolis (MG) sobre el cáncer de mama. Método: investigación cuantitativa, descriptiva, transversal con profesionales de enfermería, que habían trabajado durante al menos un año en el sistema de salud municipal. Se utilizó un cuestionario estructurado, se analizó la distribución de las variables de interés y para su presentación, se utilizó estadística descriptiva, media, frecuencia absoluta y porcentaje. Resultado: los entrevistados identificaron el conocimiento, siendo la mamografía el examen más adecuado para el diagnóstico precoz, pero se identificaron dudas en la edad y la periodicidad recomendada para el examen, y los factores limitantes más mencionados para la detección fueron la falta de conocimiento de la población y el equipo insuficiente. Conclusión: es necesario abordar el tema en el período de graduación y educación continua, inversión en la capacitación de profesionales y expansión de la prevención de enfermedades y promoción de la salud.

Palavras claves: Neoplasias de la mama; Enfermeras; Conocimiento.

\section{Cecilia Silva Santos}

Graduanda em Enfermagem, Universidade do Estado de Minas Gerais, Minas Gerais, Brasil. https://orcid.org/0000-0001-6875-582X

\section{Ana Caroline Corrêa Araujo}

Graduada em Enfermagem, Universidade do Estado de Minas Gerais, Minas Gerais, Brasil. https://orcid.org/0000-0001-9051-3720

\section{Fernanda Marcelino de Rezende e Silva}

Mestre em Enfermagem pela UFMG, Docente do Curso de Graduação em Enfermagem da Universidade do Estado de Minas Gerais (UEMG), Unidade Divinópolis. https://orcid. org/0000-0003-2236-7009

\section{Karla Amaral Nogueira Quadros}

Enfermeira. Mestre em Educação, Cultura e Organização Social pela Universidade do Estado de Minas Gerais. Minas Gerais, Brasil. Docente do Curso de Graduação em Enfermagem da Universidade do Estado de Minas Gerais (UEMG), Unidade Divinópolis. https://orcid.org/0000-0002-3750-4873

\section{Regina Consolação dos Santos}

Mestre em Ciências pela Universidade Federal de São João Del Rei, (UFSJ). Docente do Curso de Graduação em Enfermagem da Universidade do Estado de Minas Gerais (UEMG), Unidade Divinópolis. Coordenadora e Docente do Curso de Enfermagem da Universidade de Itaúna (UIT) - Minas Gerais. https://orcid.org/0000-0002-7393-3210
Silmara Nunes Andrade

Enfermeira. Doutora em Ciências da Saúde pela Universidade Federal de São João Del Rei (UFSJ). Docente do Curso de Graduação em Enfermagem da Universidade do Estado de Minas Gerais (UEMG), Unidade Divinópolis. https://orcid.org/0000-0002-1975-0827 
INTRODUÇÃO

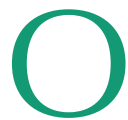
câncer de mama representa o segundo tipo de câncer mais frequente no mundo e a neoplasia mais comum em mulheres (1). Segundo estimativas do Instituto Nacional do Câncer (INCA), excetuando-se os tumores de pele não melanoma, representa o sítio mais comum na população feminina. Estimativas para cada triênio de 2020-2022 ressaltam que no Brasil são esperados 625 mil casos novos de câncer de mama, com um risco presumido de 61,61 casos a cada 100 mil mulheres ${ }^{(2)}$.

Em relação à prevalência, a situação no Brasil é semelhante ao cenário internacional. Segundo dados do relatório mundial do projeto GLOBOCAN, nos últimos anos, o câncer de mama é o mais diagnosticado na maioria dos países e também é a principal causa de morte por neoplasia em mais de 100 países ${ }^{(3,4)}$.

A elevação na incidência e mortalidade por câncer de mama no Brasil pode estar relacionada com a melhoria no diagnóstico e a qualidade das informações disponibilizadas, contudo as elevadas taxas de mortalidade podem ser atribuídas ao diagnóstico tardio dessa neoplasia devido à dificuldade de acesso da população aos serviços públicos de saúde, a baixa capacitação dos profissionais de saúde e dificuldades dos gestores municipais e estaduais em estruturar o fluxo de casos suspeitos nos diferentes níveis de atenção ${ }^{(5,6)}$.

Nos países desenvolvidos, a estratégia utilizada para a redução da mortalidade pelo câncer de mama é o rastreamento, com a finalidade de detectar o câncer em estádios iniciais. Ademais, o diagnóstico precoce e referenciamento adequado são essenciais para melhorar as taxas de cura e sobrevida. Sendo que a prevenção primária e secundária dessa neoplasia pode reduzir a incidência e a prevalência das doenças ${ }^{(1,7)}$.

Nos últimos anos, o INCA e o Mi- nistério da Saúde têm ampliado o método de comunicação para as mulheres e os profissionais de saúde, favorecendo o desenvolvimento de várias ações educativas no controle da neoplasia mamária, na Atenção Básica (AB). Assim, os profissionais que atuam na $A B$ apresentam um papel fundamental no desenvolvimento dessas ações, principalmente o enfermeiro, devido sua atividade articuladora entre os usuários e a equipe de saúde ${ }^{(8,9,10)}$.

Em relação às ações desenvolvidas pelos profissionais para detecção precoce do câncer de mama na $A B$, estudos apontam falhas em sua execução, tanto por falta de estrutura, quanto pela escassez no conhecimento teórico e técnico, como a falta de conhecimento para indicação e realização de exames clínicos e ginecológicos ${ }^{(10)}$.

Nesse contexto, a avaliação do conhecimento dos profissionais da atenção primária sobre câncer de mama, em especial os enfermeiros, é imprescindível para o delineamento de ações que contemplem capacitações em caráter contínuo e estabelecimento de um fluxo de referenciamento com o objetivo de estabelecer uma vigilância permanente.

Este estudo teve como objetivo avaliar o conhecimento relacionado ao câncer de mama de profissionais da enfermagem que atuam na atenção primária de saúde do município de Divinópolis-MG.

\section{MÉTODO}

Trata-se de um estudo quantitativo, descritivo, de delineamento transversal, realizado na cidade de Divinópolis, Minas Gerais. Divinópolis é um município de 235.977 habitantes ${ }^{(11)}$, localizado no Centro-Oeste do estado de Minas Gerais, que conta com um serviço oncológico de referência para uma população de 1.200.000 habitantes. Segundo dados do DATASUS (12), Divinópolis possui 43 unidades de atenção primária à Saúde (UAPS), distribuídas nas regiões sanitárias.
Em 2018, havia 55 enfermeiros em atividade nas UAPS do município. Todos os profissionais, de ambos os sexos, sem restrição etária e que atuassem há pelo menos um ano na rede municipal de saúde foram convidados a participar do estudo, porém apenas 48 (87,27\%) enfermeiros aceitaram, após a assinatura do Termo de Consentimento Livre e Esclarecido, os demais enfermeiros relataram falta de tempo e desinteresse.

A coleta dos dados foi realizada no período de maio a outubro de 2018, nos horários de atendimento das unidades de saúde e de acordo com a disponibilidade dos enfermeiros. Para a coleta de dados, foi utilizado um questionário estruturado constituído por 26 questões de múltipla escolha, composto por perguntas direcionadas a caracterização sociodemográfica, formação e tempo de experiência dos participantes. Além de questões referentes ao conhecimento dos enfermeiros sobre o câncer de mama, e referentes a atitudes e práticas, com relação à detecção dessa neoplasia. Este questionário foi construído tomando por base estudos realizados com metodologia semelhante à adotada neste trabalho ${ }^{(6,13)}$.

Os questionários foram entregues por meio de abordagem direta, ou seja, o pesquisador realizou pessoalmente a entrega do documento, sendo respondido por profissionais, sem qualquer interferência, permitindo que ele se expressasse livremente. Os dados foram categorizados e transcritos para uma ficha impressa. Em seguida, foi analisada a distribuição das variáveis de interesse. Para a apresentação dos mesmos, utilizou-se a estatística descritiva, média, frequência absoluta e porcentagem. Este estudo foi realizado de acordo com os princípios que orientam a realização de pesquisa envolvendo seres humanos, conforme a resolução 466/2012 ${ }^{(14)}$, e todos os procedimentos que envolveram os sujeitos foram aprovados pelo Comitê de Ética em Pesquisa em Seres Humanos da Fundação Educacional de Divi- 
Tabela 1. Distribuição de enfermeiros de acordo com as variáveis sociodemográficas, tempo de formação e experiência no serviço da APS do Município de Divinópolis (MG), 2018.

\section{Variáveis}

Número de indivíduos $(\mathrm{N}=48) \quad \%$

Faixa etária (anos)

\begin{tabular}{llc}
20 a 29 anos & 01 & 2,1 \\
30 a 39 anos & 31 & 64,6 \\
40 a 49 anos & 13 & 27,1 \\
50 a 59 anos & 03 & 6,2 \\
\hline
\end{tabular}

\section{Sexo}

\begin{tabular}{|lcc|}
\hline Feminino & 40 & 83,3 \\
\hline Masculino & 08 & 16,7 \\
\hline Estado Civil & & 35,4 \\
\hline Solteiro & 17 & 56,3 \\
\hline Casado & 27 & 8,3 \\
\hline Divorciado & 04 & \\
\hline Renda Familiar (salários) & & 29,1 \\
\hline 2 a 4 & 14 & 50,0 \\
\hline 5 a 7 & 24 & 18,8 \\
\hline 8 a 10 & 09 & 2,1 \\
\hline$>$ De 10 & 01 & \\
\hline
\end{tabular}

Tempo de Formação

\begin{tabular}{lll}
\hline 1 a 9 anos & 09 & 18,8 \\
\hline 10 a 20 anos & 34 & 70,8 \\
\hline Mais de 20 anos & 05 & 10,4 \\
\hline Tempo de atuação na atenção básica de saúde & & \\
1 a 5 anos & 08 & 16,6 \\
\hline 6 a 10 anos & 19 & 39,6 \\
\hline 11 a 15 anos & 09 & 18,8 \\
\hline Mais de 15 anos & 12 & 25,0 \\
\hline Realizou alguma especialização? & & 95,8 \\
\hline Sim & 46 & 4,2 \\
\hline Não
\end{tabular}

Fonte: Pesquisa de Campo

Tabela 2. Conhecimento sobre câncer de mama entre enfermeiros que atuam na APS do Município de Divinópolis (MG), 2018

Variáveis (Conhecimento) Número de indivíduos $(\mathrm{N}=48)$

$\%$

Há indicação de exame clínico de mamas (ECM) em mulheres assintomáticas

$\begin{array}{lcc}\text { Sim } & 47 & 97,9 \\ \text { Não } & 01 & 2,1\end{array}$

Qual o melhor período para a realização do ECM nópolis - FUNEDI parecer $\mathrm{n}^{\circ} 2.740 .529$ (CAAE: 85479318.0.0000.5115). O consentimento informado por escrito foi obtido de todos os participantes.

As limitações para realização do trabalho, foram respectivamente sobre os profissionais que se recusaram a participar da pesquisa, devido ao medo de errar as respostas do questionário, falta de tempo e porque não queriam ser avaliados por alunos.

\section{RESULTADOS}

No grupo estudado, dos 48 enfermeiros, $83,3 \%$ pertenciam ao sexo feminino, $64,6 \%$ possuíam idade inferior a 40 anos, predominância do estado civil casado e remuneração média de 5,85 salários mínimos mensais. O tempo de formado entre os enfermeiros variou de 1 a 29 anos. Quanto ao tempo de atuação na atenção básica de saúde (ABS) no Município de Divinópolis, 56,2\% dos enfermeiros referiram ter no máximo 10 anos de atuação (Tabela 01).

Sobre a qualificação desses profissionais, a maioria dos entrevistados possui mais de 10 anos de formação e apenas dois dos entrevistados não possuem algum tipo de especialização, sendo que $43,6 \%$ fizeram duas ou mais especializações, sendo "saúde da família" a mais realizada. Quanto à realização de cursos de educação continuada sobre câncer de mama apenas cerca de $40 \%$ afirmaram ter participado de cursos com essa abordagem há menos de cinco anos.

A Tabela 2 demonstra o conhecimento sobre a detecção precoce do câncer de mama entre os profissionais de enfermagem. Identificou-se que a mamografia é o exame mais indicado para a detecção precoce do câncer de mama, contudo muitos profissionais demonstraram dúvidas em relação à idade e a periodicidade recomendada para realização desse exame. Quanto os limitadores do rastreamento do câncer de mama, os fatores mais referidos entre os enfermeiros foram à insuficiência de profissionais de 


\begin{tabular}{lcc}
\hline Ovulatório & 01 & 2,1 \\
\hline Período menstrual & 01 & 2,1 \\
\hline 3 a 5 dias após a menstruação & 40 & 83,3 \\
\hline Independente & 05 & 10,4 \\
\hline Não sabe & 01 & 2,1
\end{tabular}

Qual a idade recomendada para solicitação da mamografia?

$\begin{array}{llc}30 \text { anos } & 01 & 2,1 \\ 40 \text { anos } & 20 & 41,6 \\ 50 \text { anos } & 27 & 56,3\end{array}$

Qual o exame mais indicado para o diagnóstico precoce do câncer de mama?

\section{ECM}

\begin{tabular}{lcc} 
Mamografia & 09 & 18,8 \\
\hline US mamária & 35 & 72,9 \\
\hline Ressonância magnética & 04 & 8,3 \\
O câncer de mama tem cura? & 0 & 0 \\
\hline Sim & 48 & 100 \\
Não & 0 & 0
\end{tabular}

Quais os fatores limitantes ao rastreamento do câncer de mama?

\begin{tabular}{lll}
\hline Desconhecimento da população & 38 & 79,2 \\
\hline Poucos profissionais habilitados & 11 & 22,9 \\
\hline Número de profissionais insuficientes & 14 & 29,2 \\
\hline Equipamento insuficiente & 28 & 58,3 \\
\hline Gestor público não comprometido & 18 & 37,5
\end{tabular}

Com que frequência encontra formulário de referência e contrarreferência em sua US?

\begin{tabular}{lcc} 
Sempre & 40 & 83,3 \\
\hline Frequentemente & 0 & 0 \\
Raramente & 06 & 12,5 \\
\hline Nunca & 01 & 2,1 \\
Não respondeu & 01 & 2,1
\end{tabular}

Fonte: Pesquisa de Campo

Tábela 3. Distribuição dos enfermeiros que atuam na APS, segundo a aprendizagem e educação permanente no âmbito do câncer de mama do Município de Divinópolis (MG), 2018

\section{Variáveis (Atitudes)}

Você gostaria de receber educação permanente?

Sim

Não

SMS estimula os profissionais da APS a participarem

de programa de educação permanente? saúde habilitados, a falta de equipamentos e o desconhecimento da população.

Em relação às atitudes dos enfermeiros, na detecção do câncer de mama, identificou-se uma motivação na busca de uma educação permanente em saúde, no qual todos os entrevistados relataram interesse em receber treinamento. Contudo, 25\% dos profissionais relataram que não se sentem estimulados pela Secretaria Municipal de Saúde (SMS) (Tabela 3).

Ao serem questionados sobre o conhecimento obtido na graduação para o rastreamento do câncer de mama, $87,5 \%$ responderam que houve tal abordagem na graduação. Em relação a atividades de educação continuada sobre essa neoplasia, constatou-se que 16,7\% não receberam treinamento no diagnóstico do câncer de mama pela SMS e 43,8\% receberam há mais de cinco anos, o que não atende as recomendações do SUS quanto à qualificação de recursos humanos.

Em relação as práticas dos enfermeiros na avaliação do rastreamento do câncer de mama, 77,1\% afirmaram que a solicitação da mamografia para as mulheres acima de 35 anos com alto risco é a conduta ideal. Quanto às muIheres que queixam de nódulo mamário, $77,1 \%$ relataram realizar o ECM. Durante a consulta cerca de $79 \%$ dos profissionais enfermeiros orientam sobre $\mathrm{o}$ câncer de mama, esclarecendo duvidas, repassando informações pertinentes e ressaltando a importância da investigação familiar (Tabela 4).

\section{DISCUSSÃO}

As unidades básicas de saúde são consideradas a "porta de entrada" do Sistema Único de Saúde para a população e devem reunir profissionais com capacidade de desenvolver ações educativas e preventivas, inclusive em relação ao câncer de mama, proporcionando a identificação e o encaminhamento de casos suspeitos para o 


\begin{tabular}{lcc}
\hline Sim & 36 & 75,0 \\
\hline Não & 12 & 25,0 \\
\hline $\begin{array}{l}\text { Você se sente preparado para a realização Exame Clíni- } \\
\text { Co da Mama (ECM) na unidade de saúde (US)? }\end{array}$ & \\
\hline Sim & 46 & 95,8 \\
\hline Não & 2 & 4,2 \\
\hline $\begin{array}{l}\text { Existe motivação na sua US na busca constante da } \\
\text { qualidade no atendimento? }\end{array}$ & \\
\hline Sim & 41 & 85,4 \\
\hline Não & 06 & 12,5 \\
\hline Não sabe & 01 & 2,1 \\
\hline $\begin{array}{l}\text { Participou de cursos de educação continuada sobre } \\
\text { câncer de mama? }\end{array}$ & \\
\hline Ano anterior & & \\
\hline Entre dois a cinco anos & 06 & 12,5 \\
\hline Há mais de cinco anos & 13 & 27,0 \\
\hline Nunca & 21 & 43,8 \\
\hline Não lembra & 03 & 6,3 \\
\hline Fonte: Pesquisa de Campo & 05 & 10,4 \\
\hline
\end{tabular}

serviço de referência implantado no município ${ }^{(13,15)}$.

Uma das proposições do modelo da atenção primária é a abordagem integral do indivíduo. Assim, é essencial que todos profissionais que atuam nas unidades básicas de saúde articulem e conheçam os problemas que acometem a população. E os enfermeiros são um dos principais elementos dessa estratégia, porque estão em contato direto com o usuário. Ademais, os profissionais de enfermagem apresentam o papel de educador em saúde e devem estar qualificados para desenvolver ações de prevenção e detecção precoce do câncer de mama, sendo fundamental o domínio do conhecimento sobre o assunto ${ }^{(8,13,15)}$.

Em estudo realizado no município de Ribeirão Preto (SP) evidenciou que o nível de conhecimento do profissional de enfermagem em relação ao câncer de mama estava relacionado com a as-

Tabela 4. Práticas e condutas, com relação à detecção do câncer de mama, de enfermeiros que atuam na APS do Município de Divinópolis (MG), 2018

Variáveis (Conduta)

Qual a sua conduta com uma mulher de 35 anos com alto risco para o câncer de mama?*

\begin{tabular}{llc}
\hline Retornar com 6 meses & 02 & 4,2 \\
\hline Solicitar US mamária bilateral & 06 & 12,5 \\
\hline Encaminhar ao mastologista & 09 & 18,8 \\
\hline Realizar ECM & 26 & 54,2 \\
\hline Solicitar mamografia (MMG) & 37 & 77,1 \\
\hline $\begin{array}{l}\text { Qual a sua conduta frente a uma mulher de 30 anos } \\
\text { com queixas de nódulo mamário?** }\end{array}$ & & \\
\hline Retornar com 6 meses & 0 & 0 \\
\hline Solicitar US mamária & 11 & 22,9 \\
\hline Encaminhar ao mastologista & 19 & 39,6 \\
\hline Realizar o ECM & 37 & 77,1 \\
\hline Qual a sua orientação sobre o câncer de mama?*** & & \\
\hline $\begin{array}{l}\text { Fornecer informações sobre CA mama e investigação } \\
\text { familiar }\end{array}$ & 38 & 79,2 \\
\hline Ensinar o AEM a partir dos 20 anos & 32 & 66,7 \\
\hline Importância das consultas periódicas & 33 & 68,8 \\
\hline Orientar a importância de exames complementares & 23 & 47,9 \\
\hline
\end{tabular}
$(\mathrm{N}=48)$

$\%$

Número de indivíduos 
a mamografia a partir dos 35 anos, anualmente. Sendo que nos últimos anos, o INCA e o Ministério da Saúde têm ampliado o método de comunicação para as mulheres e os profissionais de saúde. Assim, os enfermeiros devem conhecer os principais fatores de risco para o câncer de mama e os principais sinais e sintomas, que ao identificar tais indícios devem encaminhar essas mulheres imediatamente para um serviço de saúde de referência para esclarecimento e diagnóstico ${ }^{(1,6)}$.

A realização da mamografia foi $\mathrm{O}$ exame mais indicado para diagnóstico precoce, sendo a idade recomendada para sua solicitação de 50 anos (56,3\%), porém muitos profissionais demonstraram duvida quanto à idade, de forma que muitos marcaram 40 anos $(41,6 \%)$, assim o valor foi bem aproximado. E a periodicidade indicada desse exame foi bienal $(64,6 \%)$, mas com um número também expressivo do exame anual (33,3\%). Outro estudo realizado em São Paulo indicou que $72,9 \%$ dos entrevistados informaram que o período de realização da MMG é anual e 61,7\% que a idade é de 40 anos ${ }^{(16)}$.

Sobre o diagnóstico precoce, fatores e condições de risco do câncer de mama, a maioria dos profissionais demonstrou conhecimento. Quanto à realização do ECM a indicação para muIheres assintomáticas foi de 97,9\% dos entrevistados e o período indicado para realização foi de três a cinco dias após menstruação. No estudo realizado no município de Espírito Santo (RN) os entrevistados também afirmaram indicação de ECM para mulheres assintomáticas (87\%) e o mesmo período para realização do mesmo ${ }^{(17)}$.

Em relação aos fatores limitantes ao rastreamento do câncer de mama, mais de $60 \%$ dos entrevistados selecionaram dois ou mais fatores, sendo os mais recorrentes o desconhecimento da população (79,2\%), equipamento insuficiente $(58,3 \%)$ e gestor publico não comprometido $(37,5 \%)$. Com base no primei-
A realização da mamografia foi o exame mais indicado para diagnóstico precoce, sendo a idade recomendada para sua solicitação de 50 anos $(56,3 \%)$, porém muitos profissionais demonstraram duvida quanto à idade, de forma que muitos marcaram 40 anos $(41,6 \%)$, assim o valor foi bem aproximado. ro fator pode-se observar uma falha de capacitação no que diz respeito ao desenvolvimento de ações preventivas e educativas em relação ao câncer de mama, uma vez que é fator de responsabilidade do enfermeiro a educação em saúde. Em outro estudo realizado em Mossoró (RN) também foi identificado que a maioria dos entrevistados marcou duas ou mais alternativas nesta questão, e que foi identificado como mais recorrente limitador a falta de conhecimento da população $(53,1 \%$ ) e equipamentos insuficientes $(23,4 \%)^{(5)}$.

Quanto à prática e conduta do profissional de enfermagem frente à mulher de 35 anos com alto risco para câncer de mama, a metade (50\%) diz realizar duas ou mais ações, sendo as mais utilizadas a solicitação de mamografia $(77,1 \%)$ e a realização do $\operatorname{ECM}(54,2 \%)$, na maioria das vezes associadas. E quanto a conduta frente a mulher de 30 anos com queixa de nódulo mamário, 35,4\% diz realizar duas ou mais condutas, sendo a mais efetuada o $\operatorname{ECM}(77,1 \%)$.

Segundo os entrevistados quando realizam orientação para a população sobre o câncer de mama, 70,8\% utilizam dois ou mais tipos de abordagem. Desses, os mais realizados, respectivamente, são informar sobre o câncer de mama e investigação familiar, falar sobre a importância das consultas periódicas, ensinar o autoexame da mama e orientar sobre exames complementares. Mas somente $39,6 \%$ disseram realizar todos os quatro tipos que estavam contidos no questionário, o que seria ideal para uma abordagem completa sobre orientação, visando uma prevenção da doença e a promoção da saúde.

Assim, a abordagem preventiva desses fatores nas unidades básicas de saúde (UBS) e nas Estratégias de Saúde da Família (ESF) é essencial. Ressalta-se que os enfermeiros que atuam na atenção primária apresentam maior capacidade de intervenção ao nível de conhecimentos, atitudes e condutas na saúde da população, a sua função educativa deve 
aproximar os meios sociais e à cultura inter-relacionada nos mesmos. Além disso, esses profissionais podem desempenhar um papel essencial no processo de prevenção dessa patologia, através de orientações de saúde direcionada à população ${ }^{(13,15,18)}$.

\section{CONCLUSÃO}

Evidenciou-se que a maioria das questões de diagnóstico clínico, fatores e condições de risco para o câncer de mama foram respondidos pelos profissionais de enfermagem de forma correta, porém foi identificada uma lacuna no conhecimento desses profissionais, em relação à periodicidade e a idade recomendada para realização da mamografia. A educação continuada não é realizada de forma efetiva, visto que mais da metade dos entrevistados recebeu treinamento há mais de cinco anos

C6

Evidenciou-se que a maioria das questões de diagnóstico clínico, fatores e condições de risco para o câncer de mama foram respondidos pelos profissionais de enfermagem de forma correta ou não receberam, não atendendo as recomendações do SUS quanto à qualificação de recursos humanos. Foi detectada uma deficiência na promoção de saúde sobre o câncer de mama, quanto ao rastreamento do câncer de mama e uma insuficiência na educação em saúde, quanto a orientações sobre o câncer de mama. Esses achados reforçam a necessidade de abordagem do tema no período de formação acadêmica e de investimento na educação continuada, na habilitação de profissionais qualificados e de equipamentos, e também primordial, a promoção e educação em saúde para a população, motivando o autocuidado.

\section{AGRADECIMENTO}

Os autores agradecem o apoio da Fapemig.

\section{Referências}

1. Brasil. Instituto Nacional de Câncer José de Alencar Gomes da Silva. Câncer de Mama no Brasil [Internet]. Rio de Janeiro: INCA; 2019 [acesso 15 Set 2019]. Disponível em: http://www.inca.gov.br/.

2. Brasil. Instituto Nacional de Câncer José de Alencar Gomes da Silva. Estimativa 2020: Incidência de câncer no Brasil [Internet]. Rio de Janeiro: INCA; 2019 [acesso em 15 Fev 2020]. Disponível em: http://www.inca.gov. br/estimativa/2020/.

3. Bray F, Ferlay J, Soerjomataram I, Siegel RL, Torre LA, Jemal A. Global cancer statistics 2018: GLOBOCAN estimates of incidence and mortality worldwide for 36 cancers in 185 countries. CA: a cancer journal for clinicians. 2018; 68(6):394-424.

4. Ferlay J et al. Cancer today. Lyon, France: International Agency for Research on Cancer, 2018. (IARC CAncerBase, n. 15).

5. Jácome EM, Silva RM, Gonçalves MLC, Collares PMC, Barbosa IL. Detecção do Câncer de Mama: Conhecimento, Atitude e Prática dos Médicos e Enfermeiros da Estratégia Saúde da Família de Mossoró, RN, Brasil. Rev Bras de Cancerol. 2011; 57 (2):189-98.

6. Belfort LRM, Lima KM, Dutra LPF, Negro-Dellacqua M, Martins VHS, Macedo LJA. 0 papel do enfermeiro no diagnóstico precoce do câncer de mama na atenção primária. Res Soc Dev. 2019; 8(5):e 34851024.

7. Ramos MESP, Sanchez JJ, Santos LA. A ação das políticas públicas na prevenção do câncer do colo do útero e mama na atenção básica em Salvador-BA. Rev Enferm Contemp. 2016 Jan; 5(1): 5-15.

8. Lourenço TS, Mauad EC, Vieira RAC. Barreiras no rastreamento do câncer de mama e o papel da enfermagem: revisão integrativa. Rev Bras Enferm. $2016 \mathrm{Jul} ; 66(4)$ : 585-91.

9. Silva SA, Nogueira DA, Paraizo CMS, Fracolli LA. Assessment of primary health care: health professionals' perspective. Rev Esc Enferm USP. 2014; 48: $122-28$.

10. Jurado SR, Saraiva KVO, Weis M, Pereira LVRC. Sintomas depressivos em mulheres com câncer de mama submetidas à quimioterapia e radioterapia: uma revisão integrativa. Nursing (Säo Paulo), 2019; 22(253): 2967-72. 11. Brasil. Instituto Brasileiro de Geografia e Estatística. Estimativa da população 2018 [Internet]. Brasília (DF): Instituto Brasileiro de Geografia e Estatística; 2019 [acesso em 25 mar 2019]. Disponível em: https://ww2.ibge.gov. br/apps/populacao/projecao.

12. Brasil. Ministério da Saúde. DATASUS. Informações de saúde. [Internet]. Brasília (DF): Ministério da Saúde; 2019 [acesso em 10 set 2019]. Disponível em: http://tabnet.datasus.gov.br

13. Moraes DC, Almeida AM, Figueiredo EN, Loyola EAC, Panobianco MS. Rastreamento oportunístico do câncer de mama desenvolvido por enfermeiros da Atenção Primária à Saúde. Esc Enferm USP. 2016; 50(1): 14-21. 14. Brasil. Conselho Nacional de Saúde. Resolução № 466, de 12 de dezembro de 2012. Comissão Nacional de Ética em Pesquisa (CONEP/CNS/MS do CN) e pelos Comitês de Ética em Pesquisa (CEP) compondo um sistema que utiliza mecanismos, ferramentas e instrumentos próprios de inter-relação que visa à proteção dos participantes de pesquisa. Diário Oficial da União 13 jun 2013; Seção 1.

15. Zapponi ALB, Tocantins FR, Vargens OMC. 0 enfermeiro na detecção precoce do câncer de mama no âmbito da atenção primária. Rev Enferm UERJ. 2015; 23(1): 33-38.

16. Melo FBB, Marques CAV, Rosa AS, Figueiredo EN, Gutiérrez MGR. Ações do enfermeiro na detecção precoce do câncer de mama. Rev Bras Enferm. 2017; 70(6): 1183-93.

17. Fonseca DCO, Silva FT, Santos NMG, Davim, RMB. Ações na prevenção do exame do câncer de mama na consulta do enfermeiro. Rev Bras Enferm. UFPE. 2016; 10(12): 4563-71.

18. Barbosa YC, Rabêlo PPC, Aguiar MIF, Azevedo PR, Cortês LSL. Detecção precoce do câncer de mama: como atuam os enfermeiros da atenção primária à saúde? Rev. APS. 2018; 21(3): 375-86. 